\title{
Rhodium-Catalyzed Direct C-H Addition of 4,4-Dimethyl-2-oxazoline to Alkenes
}

\author{
Sean H. Wiedemann, Robert G. Bergman, ${ }^{*}$ and Jonathan A. Ellman* \\ Center for New Directions in Organic Synthesis, Department of Chemistry, University of California \\ and Division of Chemical Sciences, Lawrence Berkeley National Laboratory, Berkeley, California, \\ 94720
}

\section{Supporting Information}

I. General Experimental Procedures

$\underline{\text { Page \# }}$

II. Spectroscopic and Analytical Data

2

3 


\section{General Experimental Procedures General Experimental}

Unless otherwise noted, all reagents were obtained from commercial suppliers and degassed prior to use. Tetrahydrofuran (THF) was distilled under nitrogen from sodium/benzophenone ketyl immediately prior to use. All experiments were prepared in a $\mathrm{N}_{2}$-filled Braun inert atmosphere box. All reactions were carried out in oven-dried glassware. Thin layer chromatography was performed on Merck 60 F254 250- $\mu$ m silica gel plates. Visualization of the developed chromatogram was performed by iodine stain. Flash column chromatography was carried out using Merck 60 230-400 mesh silica gel. Organic solutions were concentrated using a rotary evaporator. All infrared (IR) spectra were recorded on a Mattson Galaxy 3000 Fourier Transform spectrometer on thin film on $\mathrm{NaCl}$ plates and only partial data are listed. ${ }^{1} \mathrm{H}$ NMR and ${ }^{13} \mathrm{C}$ NMR spectra were obtained on a Bruker AMX-400, AM-500 FT, or AMX-300 spectrometer. Unless otherwise noted, all NMR spectra were obtained in $\mathrm{CDCl}_{3}$ and referenced to residual $\mathrm{CHCl}_{3}$. Elemental analyses were performed by the University of California, Berkeley elemental analysis facility. Analytical data and synthetic protocols have been reported for the following compounds: tert-butyl-dimethyl-pent-4-enyloxy-silane, ${ }^{1}$ allyl-carbamic acid benzyl ester, ${ }^{2}\left[\mathrm{RhCl}(\mathrm{coe})_{2}\right]_{2},{ }^{3} \mathrm{PCy}_{3} \cdot \mathrm{HCl}_{1}{ }^{4} \mathrm{PCy}_{2} \mathrm{Et}^{5}{ }^{5} \mathrm{PtBu}_{2} \mathrm{Et}^{5}$. Phosphonium salts were obtained by treatment of the corresponding free base with $\mathrm{HCl}$ in ether using the procedure prescribed for the preparation of $\mathrm{PCy}_{3} \cdot \mathrm{HCl}$.

\section{General Procedure for Phosphonium Salt Screen}

To a small glass-walled bomb $(2 \mathrm{~mL})$ was added a solution of 1-hexene $(0.75 \mathrm{M})$, $\left[\mathrm{RhCl}(\mathrm{coe})_{2}\right]_{2}$ (7.5 mM with regard to monomer), phosphonium salt $(11.3 \mathrm{mM})$ and 2,6dimethoxytoluene (DMT) internal standard in THF $(0.5 \mathrm{~mL})$. Immediately prior to closing the Kontes seal $1(7.5 \mathrm{mg}, 0.15 \mathrm{M})$ was added by syringe. After heating at $45^{\circ} \mathrm{C}$ for $18 \mathrm{~h}$ the reaction was filtered through a short plug of silica, eluting with $30 \%$ ethyl acetate in hexanes $(2.5 \mathrm{~mL})$ then analyzed by GC. For this work an Agilent Technologies 6890N GC system fitted with an Agilent 7683 Series Injector and a flame ionization detector was used. Separation on an Agilent Ultra 2 column $\left(60{ }^{\circ} \mathrm{C}-2\right.$ min; 20 ${ }^{\circ} \mathrm{C} / \mathrm{min}, 150{ }^{\circ} \mathrm{C}-1 \mathrm{~min} ; 40{ }^{\circ} \mathrm{C} / \mathrm{min}, 300{ }^{\circ} \mathrm{C}-1 \mathrm{~min}$ ) gave the following retention times: 1, $2.25 \mathrm{~min}$; 2, $7.75 \mathrm{~min}$; DMT, $8.15 \mathrm{~min}$. Yields were determined relative to DMT internal standard.

\section{General Procedure for Coupling Reactions}

To a glass-walled bomb $(20 \mathrm{~mL})$ was added a solution of alkene $(5.0 \mathrm{mmol})$, $\left[\mathrm{RhCl}(\mathrm{coe})_{2}\right]_{2}(18.0 \mathrm{mg}, 0.050 \mathrm{mmol}$ with regard to monomer $), \mathrm{PCy}_{3} \cdot \mathrm{HCl}(16.0 \mathrm{mg}$, $0.050 \mathrm{mmol}), \mathrm{PCy}_{3}(7.0 \mathrm{mg}, 0.025 \mathrm{mmol})$ in THF $(6.7 \mathrm{~mL})$. Immediately prior to closing the Kontes seal 1 (99 mg, $1.0 \mathrm{mmol}$ ) was added. The reaction was heated for $18 \mathrm{~h}$ then cooled to $25^{\circ} \mathrm{C}$ and excess acid neutralized with $\mathrm{Et}_{3} \mathrm{~N}(0.5 \mathrm{~mL})$. The resulting suspension was concentrated onto silica and subjected to column chromatography eluting with $50 \%$ ether/pentane unless otherwise noted. 


\section{Spectroscopic and Analytical Data}

2-Hexyl-4,4-dimethyl-2-oxazoline (2). The reaction was performed using 1-hexene and heating at $45^{\circ} \mathrm{C}$. Chromatography afforded a clear liquid (0.136 g, 74\%). IR: 2961, 1668, 1462, 1363, 993. ${ }^{1} \mathrm{H}$ NMR (500 MHz): 3.88 (s, 2H), 2.22 (t, 2H, $\left.J=7.8 \mathrm{~Hz}\right), 1.60$ (m, 2H), 1.4-1.2 (m, 12H), $0.86(\mathrm{t}, 3 \mathrm{H}, J=6.8 \mathrm{~Hz}) .{ }^{13} \mathrm{C} \mathrm{NMR}(125 \mathrm{MHz}): 166.1,78.8,66.8$, 31.4, 28.8, 28.4, 28.1, 26.0, 22.4, 14.0. Anal. Calcd for $\mathrm{C}_{11} \mathrm{H}_{21} \mathrm{NO}$ : C, 72.08; $\mathrm{H}, 11.55 ; \mathrm{N}$, 7.64. Found: C, 72.22; H, 11.89; N, 7.49.

2-(3,3-Dimethylbutyl)-4,4-dimethyl-2-oxazoline (3). The reaction was performed as described above using 3,3-dimethylbutene and heating at $45^{\circ} \mathrm{C}$. Chromatography afforded a yellow liquid (157 mg, 86\%). IR: 2956, 1668, 1466, 1365, 1142, $982 .{ }^{1} \mathrm{H}$ NMR (500 MHz): 3.89 (s, 2H), $2.21(\mathrm{~m}, 2 \mathrm{H}), 1.54(\mathrm{~m}, 2 \mathrm{H}), 1.25(\mathrm{~s}, 6 \mathrm{H}), 0.90(\mathrm{~s}, 9 \mathrm{H})$. ${ }^{13} \mathrm{C}$ NMR (125 MHz): 166.7, 78.8, 66.8, 39.7, 30.2, 29.0, 28.4, 23.8. Anal. Calcd for $\mathrm{C}_{11} \mathrm{H}_{21} \mathrm{NO}$ : C, 72.08; H, 11.55, N, 7.64. Found: C, 71.94; H, 11.59; N, 7.85.

2-(2-Phenylethyl)-4,4-dimethyl-2-oxazoline (4). The reaction was performed using styrene and heating at $45^{\circ} \mathrm{C}$. Chromatography afforded a clear liquid $(0.075 \mathrm{~g}, 37 \%)$. IR: 2969, 1669, 1455, 1364, 1146, 990, 699. ${ }^{1} \mathrm{H}$ NMR (500 MHz): 7.29-7.18 (m, 5H), 3.90 (s, 2H), 2.95 (m, 2H), $2.56(\mathrm{~m}, 2 \mathrm{H}), 1.24$ (s, 6H). ${ }^{13} \mathrm{C}$ NMR (125 MHz): 165.1, 140.6, 128.4, 128.3, 126.2, 78.93, 66.9, 32.2, 29.9, 28.4. Anal. Calcd for $\mathrm{C}_{13} \mathrm{H}_{17} \mathrm{NO}$ : C, 76.81; H, 8.43; N, 6.89. Found: C, 76.70; H, 8.65; N, 7.13.

2-Cyclohexylmethyl-4,4-dimethyl-2-oxazoline (5). The reaction was performed using methylenecyclohexane and heating at $45^{\circ} \mathrm{C}$. Chromatography afforded a yellow oil (0.154 g, 79\%). IR: 2921, 2853, 1667, 1449, 1364, 1293, 998. ${ }^{1} \mathrm{H}$ NMR (500 MHz): 3.89 $(\mathrm{s}, 2 \mathrm{H}), 2.13(\mathrm{~d}, 2 \mathrm{H}, J=7.0 \mathrm{~Hz}), 1.71(\mathrm{~m}, 5 \mathrm{H}), 1.45-1.10(\mathrm{~m}, 10 \mathrm{H}), 0.97(\mathrm{~m}, 2 \mathrm{H}) .{ }^{13} \mathrm{C}$ NMR (125 MHz): 165.1, 78.8, 66.8, 35.8, 35.5, 33.0, 28.5, 26.2, 26.0. Anal. Calcd for $\mathrm{C}_{12} \mathrm{H}_{21} \mathrm{NO}$ : C, 73.80; H, 10.84; N, 7.17. Found: C, 74.11; H, 11.16; N, 7.26.

4,4-Dimethyl-2-(2-phenyl-propyl)-2-oxazoline (6). The reaction was performed as described above using freshly distilled $\alpha$-methylstyrene at $45^{\circ} \mathrm{C}$ except that $\left[\mathrm{RhCl}(\mathrm{coe})_{2}\right]_{2}(36.0 \mathrm{mg}, 0.100 \mathrm{mmol}$ with regard to monomer $), \mathrm{PCy} \cdot \mathrm{HCl}(32.0 \mathrm{mg}$, $0.100 \mathrm{mmol})$ and $\mathrm{PCy}_{3}(14.0 \mathrm{mg}, 0.050 \mathrm{mmol})$ in THF $(13 \mathrm{~mL})$ was used instead of the usual catalyst mixture. Chromatography, eluting with $66 \%$ ether/pentane, afforded a yellow oil (0.101 g, 46\%). IR: 2964, 1662, 1453, 1364, 993. ${ }^{1} \mathrm{H}$ NMR (300 MHz): 7.3-7.1 $(\mathrm{m}, 5 \mathrm{H}), 3.88(\mathrm{~s}, 2 \mathrm{H}), 3.19(\mathrm{~m}, 1 \mathrm{H}), 2.53(\mathrm{dd}, 1 \mathrm{H}, J=14.3,7.3), 2.47$ (dd, $1 \mathrm{H}, J=14.3$, 8.4), 1.30 (d, 3H, $J=7.0 \mathrm{~Hz}), 1.20(\mathrm{~s}, 3 \mathrm{H}), 1.12$ (s, 3H). ${ }^{13} \mathrm{C}$ NMR (75 MHz): 164.5, 145.7, 128.4, 126.8, 126.3, 78.9, 66.8, 37.3, 36.7, 28.3, 28.3, 21.4. Anal. Calcd for $\mathrm{C}_{14} \mathrm{H}_{19} \mathrm{NO}$ : C, 77.38; H, 8.81; N, 6.45. Found: C, 77.38; H, 8.86; N, 6.31.

2-Cyclohexyl-4,4-dimethyl-2-oxazoline (7). The reaction was performed using cyclohexene and heating at $45^{\circ} \mathrm{C}$. Chromatography afforded a clear liquid $(0.103 \mathrm{~g}$, 57\%). IR: 2936, 2855, 1663, 1451, 1196, 1017. ${ }^{1} \mathrm{H}$ NMR (500 MHz): 3.86 (s, 2H), 2.24 (tt, $1 \mathrm{H}, J=11.5 \mathrm{~Hz}, 3.6 \mathrm{~Hz}), 1.90(\mathrm{~m}, 2 \mathrm{H}), 1.75(\mathrm{~m}, 2 \mathrm{H}), 1.65(\mathrm{~m}, 1 \mathrm{H}), 1.40(\mathrm{~m}, 2 \mathrm{H})$, 1.25 (m, 9H). ${ }^{13} \mathrm{C}$ NMR: 169.2, 78.6, 66.5, 37.4, 29.8, 28.4, 25.8, 25.6. Anal. Calcd for $\mathrm{C}_{11} \mathrm{H}_{19} \mathrm{NO}$ : C, 72.88; H, 10.56; N, 7.73. Found: C, 72.63; H, 10.42; N, 7.76. 
4-(4,4-Dimethyl-2-oxazolin-2-yl)-butyric acid isobutyl ester (8). The reaction was performed using isobutyl vinylacetate distilled from $\mathrm{CaH}_{2}$ and heating at $105{ }^{\circ} \mathrm{C}$.

Chromatography afforded a yellow oil $(0.170$ g, 71\%). IR: 2964, 1734, 1668, 1172, 1144, 992. ${ }^{1} \mathrm{H}$ NMR (400 MHz): 3.89 (s, 2H), 3.84 (d, 2H, $\left.J=6.7 \mathrm{~Hz}\right), 2.38$ (t, 2H, $\left.J=7.5 \mathrm{~Hz}\right)$, $2.29(\mathrm{t}, 2 \mathrm{H}, J=7.4 \mathrm{~Hz}), 1.99-1.86(\mathrm{~m}, 3 \mathrm{H}), 1.25(\mathrm{~s}, 6 \mathrm{H}), 0.91(\mathrm{~d}, 6 \mathrm{H}, J=6.7 \mathrm{~Hz}) .{ }^{13} \mathrm{C}$ NMR (100 MHz): 173.1, 165.1, 78.9, 70.5, 66.9, 33.4, 28.4, 27.6, 27.3, 21.3, 19.1. Anal. Calcd for $\mathrm{C}_{13} \mathrm{H}_{23} \mathrm{NO}_{3}$ : C, 64.70; H, 9.61; N, 5.80. Found: C, 64.70; H, 9.72; N, 5.58.

3-(4,4-Dimethyl-oxazolin-2-yl)-propionic acid tert-butyl ester (9). The reaction was performed using freshly distilled tert-butyl acrylate and heating at $105^{\circ} \mathrm{C}$.

Chromatography afforded a yellow oil (0.133 g, 59\%). IR: 2972, 1730, 1671, 1366, 1251, 1148, 990. ${ }^{1} \mathrm{H}$ NMR (500MHz): $3.90(\mathrm{~s}, 2 \mathrm{H}), 2.56(\mathrm{~m}, 2 \mathrm{H}), 2.50(\mathrm{~m}, 2 \mathrm{H}), 1.43(\mathrm{~s}, 9 \mathrm{H})$, 1.24 (s, 6H). ${ }^{13} \mathrm{C}$ NMR (125 MHz): 171.4, 164.8, 80.5, 79.0, 66.9, 31.7, 28.3, 28.0, 23.4. Anal. Calcd for $\mathrm{C}_{12} \mathrm{H}_{21} \mathrm{NO}_{3}: \mathrm{C}, 63.41 ; \mathrm{H}, 9.31 ; \mathrm{N}, 6.16$. Found: C, 63.16; H, 9.39; N 6.42.

2-[5-(tert-Butyl-dimethyl-siloxy)-pentyl]-4,4-dimethyl-2-oxazoline (10). The reaction was performed using tert-butyl-dimethyl-pent-4-enyloxy-silane and heating at $105{ }^{\circ} \mathrm{C}$. Chromatography afforded a clear liquid $(0.172 \mathrm{~g}, 58 \%$ yield $)$. IR: 2932, 2859, 1669, 1463, 1254, 1102, 986, 836, 776. ${ }^{1} \mathrm{H}$ NMR (300 MHz): 3.89 (s, 2H), 3.59 (t, 2H, J=6.4 $\mathrm{Hz}), 2.24(\mathrm{t}, 2 \mathrm{H}, J=7.6 \mathrm{~Hz}), 1.63(\mathrm{~m}, 2 \mathrm{H}), 1.53(\mathrm{~m}, 2 \mathrm{H}), 1.39(\mathrm{~m}, 2 \mathrm{H}), 1.25(\mathrm{~s}, 6 \mathrm{H}), 0.88$ (s, 9H), 0.03 (s, 6H). ${ }^{13} \mathrm{C}$ NMR (75 MHz): 166.0, 78.8, 66.8, 63.0, 32.5, 28.4, 28.2, 25.9, 25.9, 25.5, 18.3, -5.3. Anal. Calcd for $\mathrm{C}_{16} \mathrm{H}_{33} \mathrm{NO}_{2} \mathrm{Si}$ : C, 64.16; H, 11.10; N, 4.68. Found: C, 63.95; H, 11.41; N, 5.00.

4,4,5,5-Tetramethyl-2-vinyl-[1,3] dioxolane. ${ }^{6}$ The condensation of pinacol and acrolein was carried out according to literature procedures. bp $90{ }^{\circ} \mathrm{C}$ at $80 \mathrm{mmHg}\left(\right.$ lit. $^{6} 80^{\circ} \mathrm{C}$ at $90 \mathrm{mmHg}$ ) IR: 2978, 1368, 1154, 1099, 978, 931. ${ }^{1} \mathrm{H}$ NMR (300 MHz): 5.83 (ddd, 1H, $J$ $=17.0,10.2,6.8 \mathrm{~Hz}), 5.41(\mathrm{~d}, 1 \mathrm{H}, J=16.5 \mathrm{~Hz}), 5.32(\mathrm{~d}, 1 \mathrm{H}, J=6.7 \mathrm{~Hz}), 5.29(\mathrm{~d}, 1 \mathrm{H}$, $10.7 \mathrm{~Hz}), 1.22(\mathrm{~s}, 12 \mathrm{H}) .{ }^{13} \mathrm{C}$ NMR (75 MHz): 137.1, 119.8, 101.0, 82.5, 24.2, 22.2.

4,4-Dimethyl-2-[2-(4,4,5,5-tetramethyl-[1,3]dioxolan-2-yl)-ethyl]-2-oxazoline (11). The reaction was performed using 4,4,5,5-tetramethyl-2-vinyl-[1,3]dioxolane and heating at $105{ }^{\circ} \mathrm{C}$. Chromatography, eluting with $75 \%$ ether/pentane, afforded a yellow oil $(0.147$ g, 58\%). IR: 2973, 1668, 1366, 1133, 966. ${ }^{1} \mathrm{H}$ NMR (500 MHz): 5.07 (t, 1H, J=4.9 Hz), $3.89(\mathrm{~s}, 2 \mathrm{H}), 2.35(\mathrm{~m}, 2 \mathrm{H}), 1.91(\mathrm{~m}, 2 \mathrm{H}), 1.25(\mathrm{~s}, 6 \mathrm{H}), 1.18(\mathrm{~s}, 12 \mathrm{H}) .{ }^{13} \mathrm{C}$ NMR $(125$ $\mathrm{MHz}): 165.5,99.7,81.8,78.9,66.8,32.4,28.3,24.2,23.1,22.1$. Anal. Calcd for $\mathrm{C}_{14} \mathrm{H}_{25} \mathrm{NO}_{3}$ : C, 65.85; H, 9.87; N, 5.49. Found: C, 65.81; H, 9.93; N, 5.29.

[3-(4,4-Dimethyl-2-oxazolin-2-yl)-propyl]-carbamic acid benzyl ester (12). The reaction was performed using allyl-carbamic acid benzyl ester and heating at $105{ }^{\circ} \mathrm{C}$. Chromatography, eluting with $100 \%$ ethyl acetate, afforded a yellow oil (0.138 g, 48\%). IR: 3329, 2966, 1717, 1664, 1531, 1250, 994. ${ }^{1} \mathrm{H}$ NMR (500 MHz): 7.4-7.2 (m, 5H), 5.21 (bs, 1H), 5.08 (s, 2H), $3.89(\mathrm{~s}, 2 \mathrm{H}), 3.26(\mathrm{~m}, 2 \mathrm{H}), 2.30(\mathrm{t}, 2 \mathrm{H}, J=7.2 \mathrm{~Hz}), 1.85(\mathrm{~m}, 2 \mathrm{H})$, 1.25 (s, 6H). ${ }^{13} \mathrm{C}$ NMR (125 MHz): 165.5, 156.4, 136.6, 128.5, 128.0, 128.0, 79.0, 67.0, 
66.5, 40.5, 28.4, 25.8, 25.6. Anal. Calcd for $\mathrm{C}_{16} \mathrm{H}_{22} \mathrm{~N}_{2} \mathrm{O}_{3}$ : C, 66.18; H, 7.64; N, 9.65. Found: C, 65.86; H, 7.82; N, 9.69.

\section{References:}

(1) Nishimura, T.; Kakiuchi, N.; Onoue, T.; Ohe, K.; Uemura, S. J. Chem. Soc., Perkin Trans. 1 2000, 1915-1918.

(2) Bischofberger, N.; Waldmann, H.; Saito, T.; Simon, E. S.; Lees, W.; Bednarski, M. D.; Whitesides, G. M. J. Org. Chem. 1988, 53, 3457-3465.

(3) Vanderent, A.; Onderdelinden, A. L. Inorg. Syn. 1990, 28, 90-92. (4) Tan, K. L.; Vasudevan, A.; Bergman, R. G.; Ellman, J. A.; Souers, A. J. Org. Lett. 2003, 5, 2131-2134.

(5) Netherton, M. R.; Fu, G. C. Angew. Chem., Int. Ed. 2002, 41, 3910-3912.

(6) Ikeda, C. K.; Braun, R. A.; Sorenson, B. E. J. Org. Chem. 1964, 29, 286-290. 